05

\title{
Фононная теплопроводность и фазовые равновесия в наночастицах системы Bi-Sb фрактальной формы
}

\author{
(c) А.В. Шишулин, ${ }^{1}$ В.Б. Федосеев, ${ }^{1}$ А.В. Шишулина ${ }^{2,3}$ \\ ${ }^{1}$ Институт металлоорганической химии РАН им. Г.А. Разуваева, \\ 603137 Нижний Новгород, Россия \\ 2 Дзержинский политехнический институт (филиал) Нижегородского государственного технического университета им. \\ Р.Е. Алексеева, \\ 606026 Дзержинск, Россия \\ ${ }^{3}$ Национальный исследовательский Нижегородский государственный университет им. Н.И. Лобачевского, \\ 603137 Нижний Новгород, Россия \\ e-mail: Chichouline_Alex@live.ru
}

(Поступило в Редакцию 19 сентября 2018 г.)

Составляющая коэффициента теплопроводности, связанная с колебаниями решетки, является одной из величин, определяющих термоэлектрическую эффективность материала. Смоделированы зависимости фазового состава и связанной с ним фононной составляющей коэффициента теплопроводности от формы наночастиц сплава $\mathrm{Bi}-\mathrm{Sb}$ эквиатомного состава с конфигурацией core-shell. Форма частицы моделируется величиной коэффициента, соответствующего степени отклонения формы частицы от сферической, или ее фрактальной размерностью. Показано, что взаимные растворимости компонентов зависят от формы наночастицы и взаимного расположения сосуществующих фаз, и в частицах сложной морфологии положению термодинамического равновесия соответствует гомогенное состояние. Гомогенизация наночастицы приводит к понижению фононной составляющей ее коэффициента теплопроводности на $70-80 \%$.

DOI: $10.21883 / J T F .2019 .04 .47311 .343-18$

\section{Введение}

Термоэлектрические материалы и приборы-преобразователи энергии на их основе в последние годы вызывают значительный интерес исследователей [1]. Основными направлениями использования термоэлектрических преобразователей энергии являются создание средств охлаждения и регулирование температуры с использованием эффекта Пельтье [2], а также генераторов электроэнергии преимущественно за счет утилизации бросового тепла [3].

Одним из подходов к созданию термоэлектрических материалов с высоким КПД является использование наноструктур с пониженной размерностью - наноразмерных пленок $[4,5]$ и квантовых проволок [6]. Однако, как отмечается в [7], с точки зрения перспективы получения коммерчески доступных высокоэффективных термоэлектриков более актуальной задачей является их создание на основе объемных нанокристаллических материалов.

Одной из основных характеристик эффективности термоэлектрического материала является величина термоэлектрической добротности $Z T \sim \sigma T / \kappa$, где $\sigma-$ электропроводимость, $\kappa-$ коэффициент теплопроводности, $T-$ рабочая температура. В первом приближении величина коэффициента теплопроводности может быть описана как совокупность двух вкладов $\kappa \approx \kappa_{c a r}+\kappa_{p h}$, где $\kappa_{c a r}$ и $\kappa_{p h}-$ вклады носителей заряда (электронов, дырок) и фононов соответственно, и, таким образом, высокие значения термоэлектриче- ской добротности $Z T$ могут быть достигнуты в первую очередь путем понижения величины фононного вклада в теплопроводность материала. Низкие величины $\kappa_{p h}$ могут быть получены, например, в твердых раствоpax [8-10].

Как отмечено в [9], твердые растворы $\mathrm{Bi}_{1-x} \mathrm{Sb}_{x}$ являются одними из наиболее эффективных низкотемпературных термоэлектрических материалов. Следует обратить внимание, однако, что высокие значения ZT и низкие $\kappa_{p h}$ (как показал расчет ab initio [9]) достижимы при $x>0.10$. Согласно имеющимся справочным данным [11], компоненты сплава $\mathrm{Bi}-\mathrm{Sb}$ образуют непрерывный ряд твердых растворов лишь при $T \geq 340 \mathrm{~K}$, при низких $T$ гомогенное состояние с $x>0.10$ является термодинамически неравновесным и сопровождается распадом твердого раствора.

Однако рассмотрение фазовых равновесий в системах нанометрового размера требует учета размерного эффекта и эффекта формы. Данные эффекты проявляются в существенном изменении растворимостей, объемных долей сосуществующих фаз и температур фазовых переходов в системах малого объема по сравнению с макросистемами, причем величины этих изменений зависят от размера системы [12-16] и геометрической конфигурации всех ее межфазных границ [17-19]. Равновесный фазовый состав, определяющий тепловые свойства наносистемы, с учетом этих эффектов может быть смоделирован методами равновесной химической термодинамики [20]. 


\section{Термодинамическая модель фазовых равновесий в наночастицах бинарных расслаивающихся твердых растворов}

Объектами моделирования являются наночастицы $\mathrm{Bi}-\mathrm{Sb}$ эквиатомного состава несферической формы при $T=100 \mathrm{~K}$. Частицы содержат то же количество вещества, что и сфера идентичного состава диаметром $d=40 \mathrm{~nm}$. Предполагается, что при распаде твердого раствора в частицах образуется единичное сферическое включение твердого раствора, окруженное слоем твердого раствора другого состава (core-shell-структура). Для описания формы наночастицы аналогично [19] введем коэффициент формы $k$, равный отношению площади поверхности рассматриваемой фигуры $A$ к площади поверхности сферы равного объема $A_{0}, k=A / A_{0}$. Геометрические характеристики core-shell-структуры описываются уравнениями:

$$
V_{j}=\sum n_{i j} V_{i}, \quad A_{c}=4 \pi\left(\frac{3}{4 \pi} V_{c}\right)^{2 / 3}, A_{s}=k \pi d^{2},
$$

где $V_{j}$ - объем фазы $j ; j=c, s$; индексам $c$ и $s$ соответствуют core- и shell-фазы; $n_{i j}$ и $V_{i}$ - число молей компонента $i$ в фазе $j$ и мольный объем компонента $i, i=1,2$; индексам 1 и 2 отвечают $\mathrm{Sb}$ и $\mathrm{Bi}$ соответственно; $A_{c}$ и $A_{s}$ - площади поверхностей coreи shell-фаз. Мольные объемы компонентов $V_{1}=18.4$, $V_{2}=21.3 \mathrm{~cm}^{3} / \mathrm{mol}$.

Условия сохранения вещества при фазовом превращении в закрытой двухкомпонентной системе связывают объем частицы, суммарное количество вещества в системе $n$, число молей каждого из компонентов $n_{i}$ и концентрации $x_{i j}$ компонентов $i$ в фазах $j$ :

$$
\begin{aligned}
\frac{1}{6} \pi d^{3} & =n_{1} V_{1}+n_{2} V_{2}, \quad n_{1}=n_{2}=0.5 n \\
n_{i} & =n_{i c}+n_{i s} \quad x_{i j}=\frac{n_{i j}}{n_{1 j}+n_{2 j}} .
\end{aligned}
$$

Критерием равновесного состояния системы является минимум функции Гиббса с учетом энергетического вклада всех границ раздела:

$$
\begin{aligned}
g= & \left(n_{1 c}+n_{2 c}\right) G\left(x_{1 c}, T\right)+\left(n_{1 s}+n_{1 s}\right) G\left(x_{1 s}, T\right) \\
& +\sigma_{s} A_{s}+\sigma_{c s} A_{c},
\end{aligned}
$$

где $\sigma_{s}, \sigma_{c s}$ - поверхностные энергии на внешней $(-s h e l l)$ и на внутренней (core-shell) границах раздела.

Функция Гиббса системы $\mathrm{Bi}-\mathrm{Sb}$ в области твердых растворов получена в [11]:

$$
\begin{aligned}
& G(x, t)=(6500-2.6 T) x(1-x) \\
& +R T(x \ln x+(1-x) \ln (1-x)) \\
& -R(2.458(1-x)(544.6-T)+2.645 x(903.7-T)),
\end{aligned}
$$

где $x$ - мольная доля $\mathrm{Sb} ; R-$ универсальная газовая постоянная.

В отсутствие надежных теоретических и экспериментальных данных о поверхностных энергиях твердых растворов величина $\sigma_{c s}$ вычислялась как $\sigma_{c s}=0.5\left(\sigma\left(x_{1 c}\right)+\sigma\left(x_{1 s}\right)\right)$, подобное приближение использовалось в [21]. Для вычисления $\sigma(x)$ использована линейная аппроксимация $\sigma(x)=\sigma_{2}(1-x)+\sigma_{1} x$, где $\sigma_{1}=0.300 \mathrm{~J} / \mathrm{m}^{2}$ и $\sigma_{2}=0.521 \mathrm{~J} / \mathrm{m}^{2}$ - поверхностные энергии чистых компонентов [19].

Высокими значениями коэффициента формы $k$ обладают простые геометрические структуры несферической конфигурации, например, для тетраэдра $k=1.49$, для конуса $k=1.52$, для прямоугольной пластины с отношением сторон $1: 1: 0.06 k=3.00$, для звездчатого икосаэдра (икосаэдра, на каждой грани которого расположен тетраэдр) $k=3.20$. Помимо этого, высокие $k$ характерны для вискеро- и дендритоподобных частиц [22], а также частиц сложной неправильной формы, для описания морфологии которых воспользуемся методами фрактальной геометрии [23,24]. Форма частиц характеризуется величиной фрактальной размерности $D$, задающей связь площади поверхности и объема $A_{s}=C V^{2 / D}$, где $C$ - численный коэффициент, полагаемый равным $4 \pi$ в дальнейших расчетах. Для простых геометрических структур $D=3.00$, для структур со сложной морфологией, примеры которых приведены на рис. $1, a$, фрактальная размерность $D<3.00$ и может не являться целым числом. В случае фрактальных частиц коэффициент формы становится функцией их объема и фрактальной размерности

$$
k(V, D)=\frac{V^{2 / D}}{(3 V / 4 \pi)^{2 / 3}} .
$$

Частицам, объем которых равен объему сферы диаметром $40 \mathrm{~nm}$, с фрактальными размерностями 2.90, $2.75,2.60$ отвечают коэффициенты формы 1.23, 1.72 и 2.51 соответственно.

\section{Результаты моделирования и обсуждение}

Функция Гиббса для core-shell-структуры (3) имеет два минимума, различающиеся тем, какой из сосуществующих твердых растворов находится в положении shell-фазы. При макроскопическом размере системы, где энергетический вклад границ раздела мал, состав сосуществующих фаз для обоих минимумов соответствует справочным данным [11]. В системе нанометрового размера значительный вклад энергии межфазных границ приводит к смещению минимумов, в результате чего равновесные составы и объемы сосуществующих фаз в этих двух конфигурациях отличаются от справочных значений для макросистем, различаются между собой и зависят от объема и формы наночастицы $[16,19]$. 

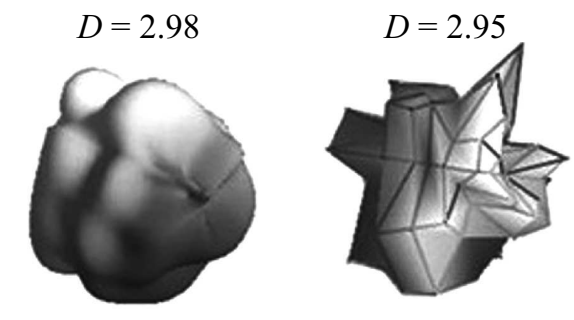

$D=2.91$
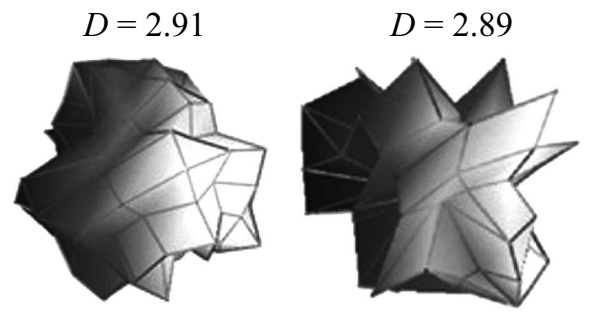

$a$

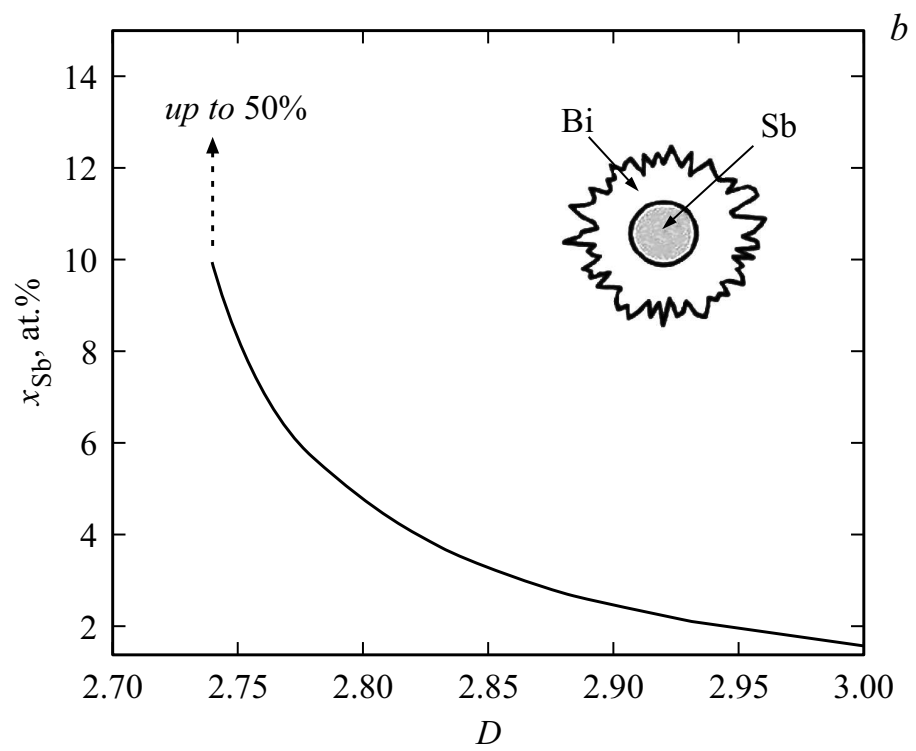

Рис. 1. $a-$ примеры фрактальных структур с различными $D ; b-$ зависимость растворимости $\mathrm{Sb}$ в Ві для конфигурации, где core-фаза образована твердым раствором на основе $\mathrm{Sb}$. При $D<2.74$ положению термодинамического равновесия соответствует гомогенное состояние частицы с $50 \% \mathrm{Sb}$.
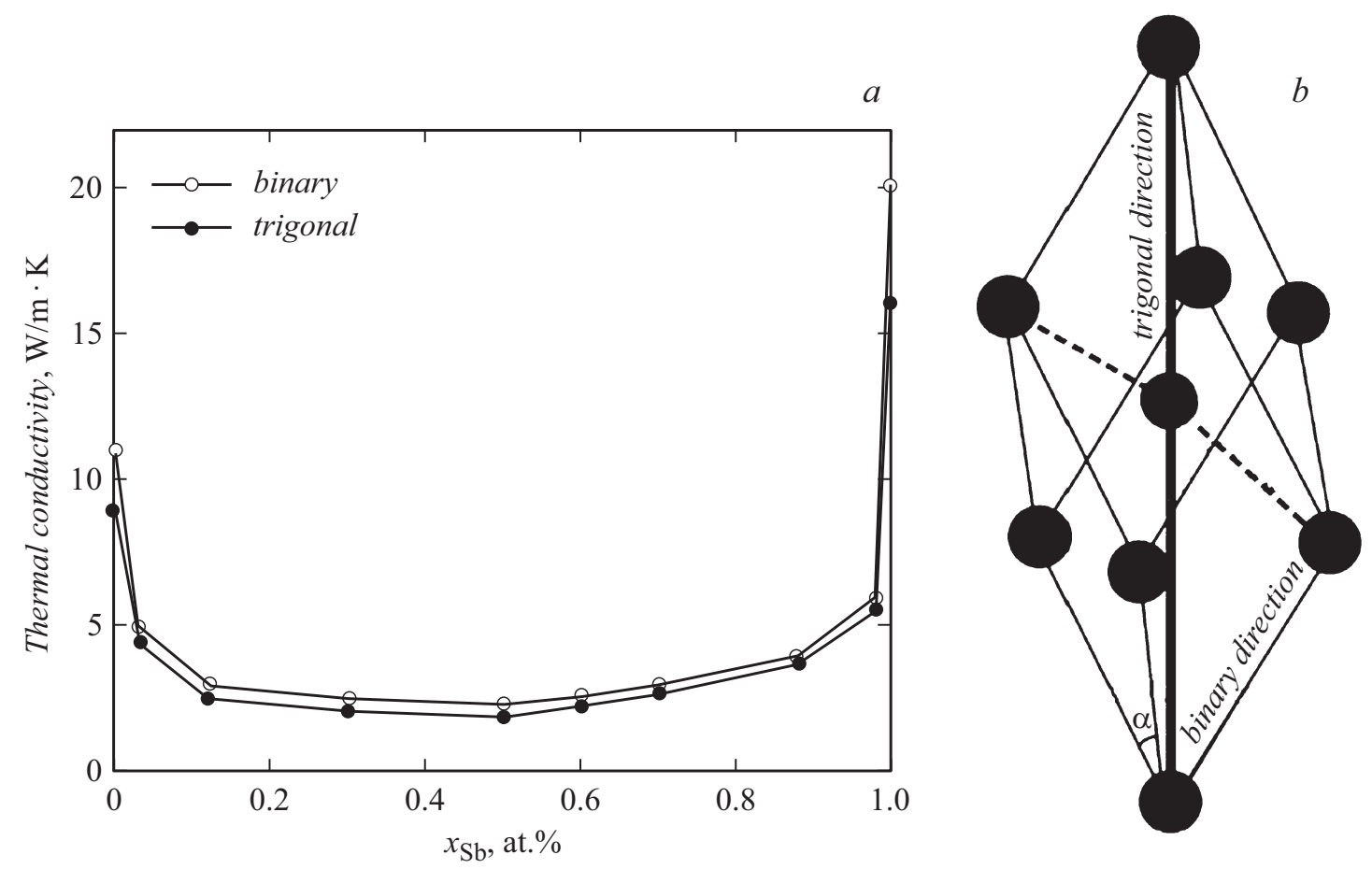

Рис. 2. $a-$ - фононная теплопроводность твердых растворов системы $\mathrm{Bi}-\mathrm{Sb}$, полученная ab initio [9] для двух различных кристаллографических направлений; $b$ - кристаллическая структура Вi и $\mathrm{Sb}$. Величина угла $\alpha$ для $\mathrm{Bi}$ составляет $57^{\circ} 30^{\prime}$, для $\mathrm{Sb}-57^{\circ} 84^{\prime}$.

Фазовые диаграммы наносистем в различных конфигурациях не совпадают ни между собой, ни с фазовой диаграммой для макросистемы. Для конфигурации, где core-фаза образована твердым раствором на основе $\mathrm{Sb}$, уменьшение $D$ (,усложнение“ формы) с 3.00 до 2.74 сопровождается существенным ростом растворимости $\mathrm{Sb}$ в Bi c $\sim 1.6$ до $\sim 10$ at.\% (рис. 1, $b$ ). Объемная доля coreфазы уменьшается с $\sim 47$ до $\sim 41 \%$, растворимость Ві в $\mathrm{Sb}$ незначительно снижается (в пределах 1 at.\%). При $D<2.74$ для рассматриваемой конфигурации минимум, соответствующий гетерогенному состоянию, исчезает, и положению термодинамического равновесия соответ- 

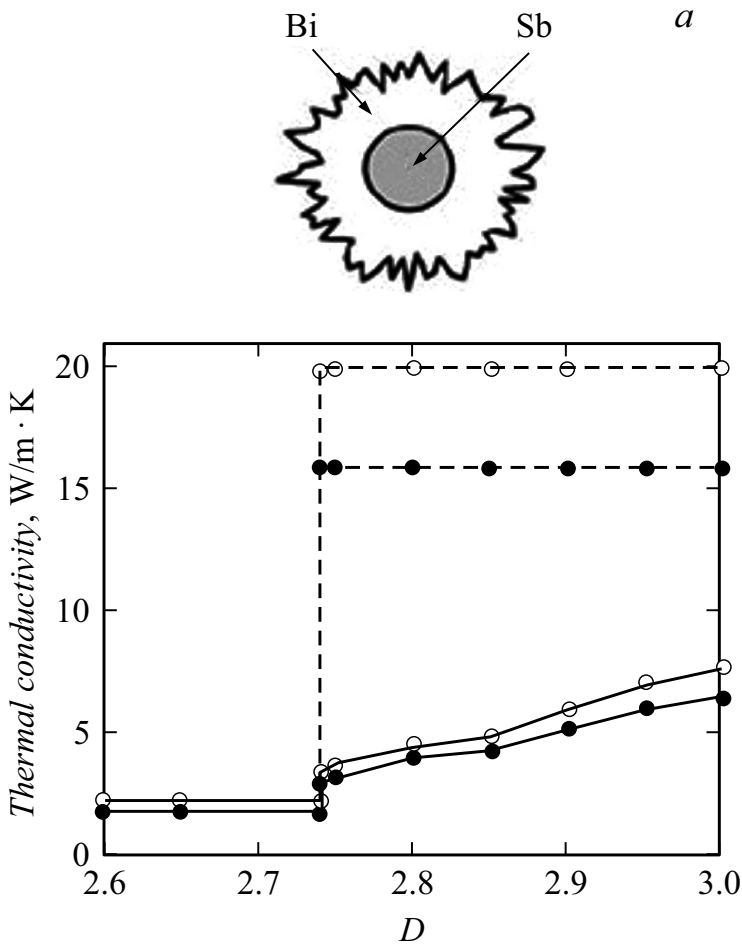

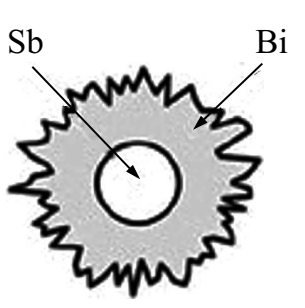

$b$

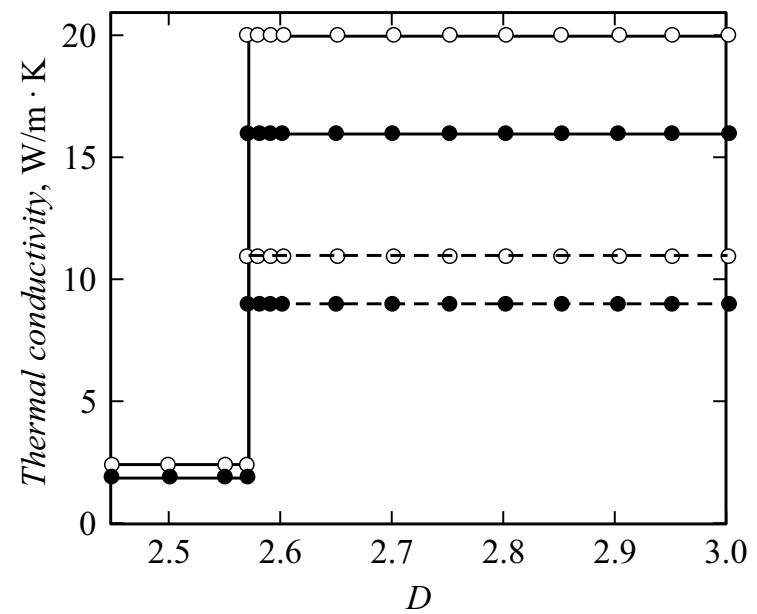

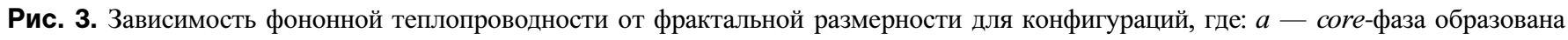
твердым раствором на основе $\mathrm{Sb} ; b-$ core-фаза образована твердым раствором на основе Ві. Сплошные линии соответствуют теплопроводности shell-фазы, штриховые - теплопроводности core-фазы. Обозначения кристаллографических направлений (закрашенные и незакрашенные маркеры) соответствуют таковым на рис. 2.

ствует гомогенная частица, содержащая $50 \% \mathrm{Sb}$ (вертикальная штриховая линия на рис. $1, b)$. Для конфигурации, где core-фаза образована твердым раствором на основе $\mathrm{Bi}$, уменьшение $D$ с 3.00 до 2.57 сопровождается незначительными (в пределах 1 at.\%) ростом растворимости $\mathrm{Sb}$ в $\mathrm{Bi}$, падением растворимости $\mathrm{Bi}$ в $\mathrm{Sb}$ и незначительным ростом доли объема, занимаемого core-фазой. Для данной конфигурации гомогенное состояние является единственным термодинамически равновесным при $D<2.57$.

Рост растворимости $\mathrm{Sb}$ в $\mathrm{Bi}$ (рис. 1, $b$ ) и падение объемной доли core-фазы являются следствием понижения системой собственной поверхностной энергии путем переноса вещества из внутренней фазы и уменьшения протяженности внутренней границы раздела, а также путем преимущественного обогащения shell-фазы компонентом с меньшей поверхностной энергией $(\mathrm{Sb})$, что приводит к уменьшению энергии внешней границы. Как показано в $[15,16,19]$, увеличение $k$ сопровождается снижением верхней критической температуры растворения (ВКТР) на фазовой диаграмме наносистемы; гомогенное термодинамически равновесное состояние соответствует фрактальным размерностям, при которых ВКТР падает ниже рассматриваемой температуры.

Зависимость фононной теплопроводности раствора $\mathrm{Bi}-\mathrm{Sb}$ от состава, полученная ab initio для двух кристаллографических направлений [9], приведена на рис. 2,a (сами направления изображены на рис. 2, b). Воспользовавшись полученными выше термодинамическими оценками равновесного фазового состава наночастиц различной фрактальной размерности и имеющимися данными, рассмотрим влияние геометрических факторов на фононную теплопроводность наночастиц. Следует отметить, что результаты настоящей работы могут быть несколько уточнены путем дополнительного учета влияния вакансий и протяженных дефектов типа дислокаций на фононную теплопроводность наночастиц [25].

Для конфигурации, где core-фаза образована твердым раствором на основе $\mathrm{Sb}$, уменьшение $D$ с 3.00 до 2.74 приводит к снижению фононной теплопроводности shell-фазы на $\sim 56 \%$ (см. рис. 3, а для двух различных кристаллографических направлений). Теплопроводность core-фазы остается практически неизменной. При $D<2.74$ термодинамически равновесным состоянием становится гомогенное с 50 at.\% Sb, что приводит к скачкообразному падению теплопроводности. Величина скачка составляет $\sim 71 \%$ по сравнению с теплопроводностью shell-фазы при $D=3.00$ и $\sim 88 \%$ по сравнению с теплопроводностью core-фазы.

Для конфигурации, где core-фаза образована твердым раствором на основе $\mathrm{Bi}$, уменьшение $D$ с 3.00 до 2.57 не сопровождается существенным изменением теплопроводности core- и shell-фаз. Но при $D<2.57$ теплопроводность скачкообразно меняется на $\sim 79 \%$ по сравне- 
нию с теплопроводностью core-фазы при $D=3.00$ и на $\sim 88 \%$ по сравнению с теплопроводностью shell-фазы.

Таким образом, в наночастицах сложной формы рост взаимной растворимости компонентов приводит к существенному (на 70-80\%) снижению фононной теплопроводности. Форма реальных наночастиц, соответствующая низким фрактальным размерностям, не является редким случаем (см., например, [22,26,27]). Высокие величины коэффициента формы $k$ также достижимы в вытянутых вискероподобных структурах (величина $k$, соответствующая фрактальным размерностям $D<2.57$, отвечает сплюснутому эллипсоиду вращения с отношением осей $a / b>5$; синтез подобного рода структур производился авторами [22]), что позволяет ожидать более низких значений $\kappa_{p h}$ для протяженных в одном направлении частиц.

Аналогичных закономерностей следует ожидать и при рассмотрении поликристаллических сплавов ограниченно взаимно растворимых компонентов, где в роли внешней границы раздела выступает граница зерна. Величины энергий границ зерен могут быть получены в рамках различных подходов (например, $[28,29]$ ), а иные варианты размещения сосуществующих фаз, связанные, например, с зарождением новой фазы на границе зерна, потребуют незначительной модификации уравнений (1)-(3). В этом случае также ожидаемы преимущества структур с неравноосными кристаллитами. При этом в поликристаллических термоэлектрических сплавах следует ожидать бо́льших величин $Z T$ и еще меньших $\kappa_{p h}$ из-за дополнительного рассеяния фононов на границах зерен, туннелирования носителей между нанозернами и энергетической фильтрации носителей [30-32].

Введенный выше коэффициент формы $k$ является непрерывным параметром, что позволяет обобщить различные варианты изохорических преобразований системы, включая плавные деформации. При этом деформация поликристаллического материала, сопровождающаяся изменением формы межзеренных границ, может приводить к значительным изменениям его термоэлектрических свойств.

\section{Заключение и выводы}

Проведенный методами равновесной химической термодинамики анализ фазовых равновесий в наночастицах расслаивающегося твердого раствора $\mathrm{Bi}-\mathrm{Sb}$ и связанной с ними фононной составляющей коэффициента теплопроводности приводит к следующим выводам:

1) в частицах расслаивающихся твердых растворов нанометрового размера равновесный состав и количество сосуществующих фаз являются функцией геометрических параметров наночастицы. При этом для частиц сложной морфологии (с формой, сильно отличающейся от сферической) распад твердого раствора термодинамически невыгоден, и устойчивым является любой состав частиц вплоть до эквиатомного. При этом гомогенизация ожидаема во множестве геометрически весьма разнородных структур, характеризуемых одними и теми же коэффициентами формы и фрактальными размерностями;

2) изменение равновесного состава сосуществующих фаз в зависимости от геометрических свойств частицы позволяет ожидать существенного изменения фононной составляющей ее коэффициента теплопроводности. При гомогенизации падение вклада колебаний решетки в коэффициент теплопроводности является весьма существенным (до 4-5 раз).

Работа выполнена при поддержке РФФИ (проект № 18-08-01356), РФФИ и Правительства Нижегородской области (проект № 18-43-520039).

\section{Список литературы}

[1] Du Y., Xu J., Paul B., Eklund P. // Appl. Mater. Today. 2018. Vol. 12. P. 366-388. DOI: 10.1016/j.apmt.2018.07.004

[2] Hu J.-Z., Liu B., Zhou J., Li B., Wang Y. // Jpn. J. Appl. Phys. 2018. Vol. 57. P. 71801-71806. DOI: $10.7567 / J J A P .57 .071801$

[3] Din L.C., Meyerheinrich N., Tan L., Rahaoui K., Jain R., Akbarzadeh A. // Energy Procedia. 2018. Vol. 110. P. 32-37. DOI: 10.1016/j.egypro.2017.03.101

[4] Li Z., Miao N., Zhou J., Sun Z., Liu Z., Xu H. // Nano Energy. 2018. Vol. 43. P. 285-290.

DOI: 10.1016/j.nanoen.2017.11.043

[5] Ерофбеева И.В., Дорохин М.В., Лесников В.П., Кузнецов Ю.М., Здоровейщев А.В., Питиримова Е.А. // ФТП. 2017. Т. 51. Вып. 11. С. 1456-1461. [Erofeeva I.V., Dorokhin M.V., Lesnikov V.P., Kuznetsov Y.M., Zdoroveyshchev A.V., Pitirimova E.A. // Semiconductors. 2017. Vol. 51. N 11. P. 1403-1408.] DOI: $10.1134 / \mathrm{S} 1063782617110112$

[6] Caballero-Calero O., Martín-González M. // Scripta Mater. 2016. Vol. 111. P. 54-57.

DOI: $10.1016 /$ j.scriptamat.2015.04.020

[7] Булат Л.П., Новотельнова А.В., Тукманова А.С., Ережеп Д.Е., Освенский В.Б., Сорокин А.И., ПшенайСеверин Д.А., Аимонтас С. // ЖТФ. 2017. Т. 87. Вып. 4. C. 484-592. [Bulat L.P., Novotel'nova A.V., Tukmanova A.S., Yerezhep D.E., Osvenskii V.B., Sorokin A.I., PshenaiSeverin D.A., Asmontas S. // Tech. Phys. 2017. Vol. 62. N 4. P. 604-612.] DOI: 10.1134/S1063784217040053

[8] Otsuka M., Homma R., Hasegawa Y. // J. Electron. Mater. 2017. Vol. 46. N 5. P. 2752-2764. DOI: $10.1007 / \mathrm{s} 11664-016-4955-\mathrm{x}$

[9] Lee S., Esfarjani K., Mendoza J., Dresselhaus M.S., Chen G. // Phys. Rev. B. 2014. Vol. 89. P. 85206-85215. DOI: 10.1103/PhysRevB.89.085206

[10] Петрова Н.С., Данилов В.А., Бойков Ю.А., Кузнецова В.С., Новиков С.В. // ЖТФ. 2018. Т. 88. Вып. 7. C. 1057-1059. [Petrova N.S., Danilov V.A., Boikov Y.A., Kuznetsova V.S., Novikov S.V. // Tech. Phys. 2018. Vol. 63. N 7. P. 1026-1028.] DOI: $10.1134 / \mathrm{S} 106378421807023 \mathrm{X}$

[11] Быков М.А., Воронин Г.Ф., Мухамеджсанова Н.М. Прямые и обратные задачи химической термодинамики. Новосибирск: Наука, 1987. С. 30.

[12] Sim K., Lee J. // J. Alloy. Compd. 2014. Vol. 590. P. 140-146. DOI: 10.1016/j.jallcom.2013.12.101 
[13] Sopoušek J., Vřešt’al J., Pinkas J., Broz P., Buršík J., Styskalik A., Skoda D., Zobak A., Lee J. // CALPHAD. 2014. Vol. 45. P. 33-39. DOI: 10.1016/j.calphad.2013.11.004

[14] Monji F., Jabbareh M.A. // CALPHAD. 2017. Vol. 58. P. 1-5. DOI: $10.1016 /$ j.calphad.2017.04.003

[15] Федосеев В.Б., Шишулин А.В., Титаева Е.К., Федосеева E.H. // ФТТ. 2016. Т. 58. Вып. 10. С. 2020-2025. [Fedoseev V.B., Shishulin A.V., Titaeva E.K., Fedoseeva E.N. // Phys. Solid State. 2016. Vol. 58. N 10. P. 2095-2100.] DOI: $10.1134 / \mathrm{S} 1063783416100152$

[16] Шишулин А.В., Федосеев В.Б. // Неорган. матер. 2018. T. 54. № 6. C. 574-578. [Shishulin A.V., Fedoseev V.B. // Inorg. Mater. 2018. Vol. 54. N 6. P. 546-549.] DOI: $10.1134 / \mathrm{S} 0020168518050114$

[17] Guisbiers G., Mendoza-Pérez R., Bazán-Díaz L., MendozaCruz R., Velázquez-Salazar J.J., Yakamán M.J. // J. Phys. Chem. 2017. Vol. 121. N 12. P. 6930-6939.

DOI: 10.1021/acs.jpcc.6b09115

[18] Cui M., Lu H., Jiang H., Cao Z., Meng X. // Sci. Rep. 2017. N 7. P. $1-10$. DOI: $10.1038 /$ srep41990

[19] Федосеев В.Б., Шишулин А.В. // ФТТ. 2018. Т. 60. Вып. 7. C. 1382-1388. [Fedoseev V.B., Shishulin A.V. // Phys. Solid State. 2018. Vol. 60. N 7. P. 1398-1404.] DOI: $10.1134 / \mathrm{S} 1063783418070120$

[20] Федосеев В.Б., Федосеева Е.Н. // ЖФХ. 2014. Т. 88. № 3. C. 446-451. [Fedoseev V.B., Fedoseeva E.N. // Russ. J. Phys. Chem. A. 2014. Vol. 88. N 3. P. 436-441.] DOI: $10.1134 / \mathrm{S} 0036024414020083$

[21] Hourlier D., Perrot P. // Mater. Sci. Forum. 2010. Vol. 653. P. 77-85. DOI: 10.4028/www.scientific.net/MSF.653.77

[22] Князев А.В., Буланов Е.Н., Власова Е.В. // Перспективные материалы. 2012. № 2. С. 42-45. [Knyazev A.V., Bulanov E.N., Vlasova E.V. // Inorg. Mater. Appl. Res. 2012. Vol. 3. N 5. P. 417-420.] DOI: 10.1134/S2075113312050073

[23] Федосеев В.Б. // Письма о материалах. 2012. Т. 2. C. $78-83$.

[24] Kalinin S.V., Gorbachev D.L., Borisevich A.Yu., Tomashevitch K.V., Vertegel A.A., Markworth A.J., Tretyakov Yu.D. // Phys. Rev. E. 2000. Vol. 61. N 2. P. 1189-1194. DOI: 10.1103/PhysRevE.61.1189

[25] Глазков В.Н. Кинетические и электрические явления в твердых телах и металлах. М.: МФТИ, 2015. 28 с.

[26] Bose A., Schuh C.A., Tobia J.C., Tuncer N., Mykulowycz N.M., Preston A., Barbati A.C., Kernan B., Gibson M.A., Krause D., Brzezinski T., Schroers J., Fulop R., Myerberg J.S., Sowerbutts M., Chiang Y.-M., Hart A.J., Sachs E.M., Lomeli E.E., Lund A.C. // Int. J. Refract. Met. 2018. Vol. 73. P. 22-28. DOI: $10.1016 /$ j.jirmhm.2018.01.019

[27] Гусев А.И., Садовников С.И. // Письма в ЖЭТФ. 2017. T. 106. № 7. C. 434-439. [Gusev A.I., Sadovnikov S.I. // JETP. Lett. 2017. Vol. 106. N 7. P. 454-459.] DOI: $10.1134 / \mathrm{S} 0021364017190080$

[28] Marcinkowski M.J. // Phil. Mag. A. 1968. Vol. 145. N 17. P. 159 - 168. DOI: $10.1080 / 14786436808218189$

[29] Tyson W., Miller W. // Surf. Sci. 1977. Vol. 62. N 267. P. 267-276. DOI: 10.1016/0039-6028(77)90442-3

[30] Bulat L.P., Bublik V.T., Drabkin I.A., Karataev V.V., Osvenskii V.B., Parkhomenko Yu.N., Pivovarov G.I., PshenaiSeverin D.A., Tabachkova N.Yu. // J. Electron. Mater. 2010. Vol. 39. N 9. P. 16504-41653.

DOI: $10.1007 / \mathrm{s} 11664-010-1250-0$

[31] Bulat L.P., Drabkin I.A., Karatayev V.V., Osvenskii V.B., Parkhomenko Yu.N., Pshenay-Severin D.A., Sorokin A.I. // J. Electron. Mater. 2014. Vol. 43. N 6. P. 2121-2126. DOI: $10.1007 / \mathrm{s} 11664-014-2988-6$
[32] Goldsmid H.J. Introduction to thermoelectricity. Berlin: Springer-Verlag, 2016. P. 74-76. DOI: $10.1007 / 978-3-642-00716-3$ 